\title{
PERCEPCIONES DE LA SALUD GENERAL, ORAL Y VISUAL DEL ADULTO MAYOR QUE ASISTE A LAS CLÍNICAS DE LA UNIVERSIDAD SANTO TOMÁS, FACTORES QUE INFLUENCIAN SU CALIDAD DE VIDA *
}

\author{
${ }^{1}$ Gloria Cristina Aránzazu M., ${ }^{2}$ Herman Boada C., ${ }^{3}$ Carmen Alodia Martínez L., ${ }^{4}$ María Mónica Uribe M. \\ ${ }^{1}$ Odontóloga U. Santo Tomás, Especialista en Patología Oral y Medios Diagnósticos U. El Bosque, Docente U. Santo Tomás. \\ ${ }^{2}$ Odontólogo Integral U. Nacional de Colombia, Docente U. Santo Tomás. ${ }^{3}$ Odontóloga U. Santo Tomás, Especialista en Ortopedia Maxilar \\ U. Antonio Nariño, Docente U. Santo Tomás. ${ }^{4}$ Optometra U. La Salle, Docente U. Santo Tomás.
}

Autor responsable de correspondencia: Gloria Cristina Aránzazu Moya

Dirección de correo electrónico: aranzazugloria@yahoo.es

\section{RESUMEN}

Objetivo: Determinar las percepciones de salud del adulto mayor y los factores que influencian su calidad de vida.

Materiales y Métodos: Se realizó un estudio descriptivo que analizó la relación de la percepción de salud con variables sociodemográficas, de salud general, enfermedades crónicas así como factores orales y visuales, necesidad de tratamiento. Los datos se obtuvieron por encuestas, se describieron las características sociodemográficas y las variables relacionadas con conocimientos y percepciones sobre la calidad de vida del adulto mayor. Se aplicaron pruebas no paramétricas, las asociaciones se evaluaron con la prueba de $\mathrm{X}^{2} \mathrm{y}$ test exacto de Fisher. Todo el análisis consideró un nivel de significancia $\mathrm{p}=0.05$, la base de datos se elaboró en Excel y el análisis definitivo en STATA 6.0.

Resultados: La población de 46 hombres y 60 mujeres con edades que variaron entre 56 - 91 . Sesenta y seis personas tenían una perspectiva positiva de su salud general, y 48 han cambiado de actividades por culpa de ella. Con respecto a la salud visual se encontró una percepción negativa en 56 (54.3\%). Respecto a la salud bucal, la percepción de la salud fue positiva para 50 (47.1\%), y negativa para 56 (52.8\%). Se evidenció que el factor que más afecta la calidad de vida es dejar actividades tanto generales, visuales y dejar alimentos con $\mathrm{P}=0.0001$.

Conclusiones: La condición que más afecta la calidad de vida es el tener que dejar actividades tanto por salud general, oral y visual. Las percepciones fueron más negativas en el género femenino. [Aranzazu GC, Boada H, Martírnez CA, Mantilla M. Percepciones de la salud general, oral y visual del adulto mayor que asiste a las clínicas de la Universidad Santo Tomás, factores que influencian su calidad de vida, 2006. Revista Ustasalud Odontología 2007; 6: 87 -95]

Palabras clave: Percepciones de salud, Calidad de vida, Adulto mayor.

\section{PERCEPTIONS OF THE GENERAL, ORAL AND VISUAL HEALTH IN THE ELDERLY THAT ATTEND THE DENTAL CLINICS OF THE SANTO TOMAS UNIVERSITY, FACTORS THAT INFLUENCE THEIR QUALITY OF LIFE}

\begin{abstract}
Objective: To determine the perceptions of the elderly health and factors that influences their quality of life.

Material and Methods: A descriptive study was done to analyze the relationship of the perception of health with sociodemographics, general health, chronic illnesses as well as oral and visual factors, necessity of treatment self perception and clinically perceived and last medical visit variables. Non parametric tests were applied; the possible associations among variables measures in nominal and ordinal scale were evaluated applying the test of X2 and exact test of Fisher. The whole analysis considers a significant level p $=0.05$, the database was elaborates in Excel and the definitive analysis in STATA 6.0.

Results: The population was composed by 46 males and 60 women with mean age of 68.6 years-old. Sixty six individuals have a positive perception of its general health and forty eight have changed activities for blame of it. The negative perception of their visual health was found in 56 (54.3) and their quality of life has been affected by their visual health on the average 3.7. The quality of life has been affected by its oral health in 3.6 on the average being negative this perception. It was evidenced that the factor that more affects the quality of life is leaving activities, leaving some foods and visual with $\mathrm{P}=0.0001$.

Conclusions: The condition that affects the quality of life more is to have to leave activities by general, oral and visual health as much. The perceptions were more negative in the female.
\end{abstract}

Key words: Perceptions of health, Quality of life, Elderly.

Recibido para publicación: 16 de octubre de 2007. Aceptado para publicación: 29 de noviembre de 2007.

*Grupo de Investigación SIBAM. 


\section{INTRODUCCIÓN}

Mundialmente, la población mayor de 60 años aumenta aproximadamente en un millón cada mes y la expectativa de vida ha aumentado de 64 a 74 años. En 1991, en Colombia había dos millones de personas mayores de 60 años y en el 2005 se calculó que el $6.31 \%$ de la población colombiana fueron adultos mayores (2.655.910 aproximadamente); se estima que para el 2025 haya 7 millones, equivalentes al $13 \%$ de la población total. ${ }^{1-3}$

En el documento Estrategia Internacional para la Acción (2002) sobre el envejecimiento, se plantea la necesidad de disponer de información local fiable y de identificar los mayores problemas para así poder dar respuestas políticas a tiempo, lo que hace necesaria una agenda mundial de investigación sobre el envejecimiento que sirva para dar respuestas y soluciones que garantice el éxito de la estrategia. ${ }^{4}$

El interés de la gerontología en los últimos años está dirigido a conocer, y más que esto, a descifrar las desconocidas interrelaciones de las condiciones y el estilo de vida de los ancianos, y a determinar cómo influye su forma de vida, tanto individual como colectiva. El objetivo es mantener la calidad de vida en esta etapa del ciclo vital, especialmente para preservar su autonomía e independencia ya que estos dos tópicos son los que aumentan los costos en su atención.

Es evidente que el adulto mayor maneja una serie de mitos y conceptos errados acerca de su salud que no lo motivan a asistir a la consulta quizás por esto su calidad de vida se deteriora notablemente. Por ejemplo, el pensar que es normal ser un adulto mayor sin dientes genera mala alimentación, poca socialización y comunicación; que eso a nadie le importa, o que ser anciano se asocie con poca visión, audición, dolor, cansancio o falta de dientes ocasiona cambios en el estilo de vida, abandono de sus ocupaciones habituales y poca socialización. ${ }^{1}$ Estas situaciones se pueden cambiar a través de un adecuado diagnóstico de la situación y mediante la educación del adulto mayor y sus cuidadores. ${ }^{5}$

Se ha encontrado una feminización en los grupos de mayor edad y estos grupos reportan peor percepción de su estado de salud y calidad de vida con una alta prevalencia de problemas crónicos. $^{6}$
La salud oral puede afectar la salud general en diferentes formas, puede causar sufrimiento y dolor así como también dificultad en hablar, masticar y deglutir. La salud visual produce cambios a nivel comportamental debido a que la persona con disminución de su visión debe adaptar su labor diaria hasta donde su capacidad visual le permita. Ettinger hace referencia a que la condición dental restringe la actividad diaria en un $7,4 \%$ siendo mayor que los problemas visuales los cuales la restringen en un $1,6 \% .^{7}$

Esas complicaciones a nivel oral pueden complicar la toma de medicamentos usados para el tratamiento de enfermedades sistémicas. Además, el tratamiento de enfermedades sistémicas puede complicarse por infecciones bacterianas de origen bucal. También existen asociaciones entre salud oral y salud general y bienestar. Por ejemplo, la pérdida de autoestima se asocia con pérdida de dientes y enfermedades como caries dental y enfermedad periodontal no tratadas. Aunque los problemas de salud oral, usualmente, no causan la muerte, el cáncer oral produce cerca de ocho mil muertes cada año, y más de la mitad de esas muertes ocurren en personas de 65 años o más. ${ }^{8}$

Los adultos mayores se configuran como una categoría independiente del resto de la sociedad, separados como grupo con características propias. De esta manera, se determinan tres conceptos: A) Vejez cronológica, que se define por haber cumplido 65 años, también determinada por la edad de retiro laboral. B) Vejez Funcional, se relaciona como sinónimo de incapaz o limitado. Es un concepto erróneo, en donde se debe luchar contra la idea de que el adulto mayor es funcionalmente limitado, se acepta que hay limitaciones pero que no lo imposibilitan para desarrollar una vida plena, como persona que vive y se desarrolla no sólo en lo físico, sino en lo psíquico y en lo social. C) Vejez, etapa vital. El transcurso del tiempo produce efectos en la persona, es una etapa más de la experiencia humana.

Comprender a la persona adulta mayor es esencial para el clínico ${ }_{2}$ ya que en el campo de la salud, es fundamental comunicarse en forma efectiva con ellos para lograr una mejor comprensión del complejo mundo de los signos y síntomas de las enfermedades. Una buena información es muy importante para lograr esa comunicación, y conocer de esta forma la percepción que los individuos adultos mayores tienen sobre su propia salud. Esta percepción se ha asociado a indicado- 
res tales como factores sociodemográficos, capacitación o conocimiento de las enfermedades y necesidad de tratamiento. ${ }^{9}$

Es importante analizar el impacto de la población creciente de la tercera edad sobre la prestación de servicios de salud (médicos, odontológicos y visuales) y conocer cómo el adulto mayor se siente acerca de su salud (auto percepción) y del impacto (valor) que tiene en su vida diaria en los aspectos fisiológicos, sociales, psicológicos y en su calidad de vida, con el fin de mejorar e instaurar estrategias para la interacción con este grupo poblacional.

Se ha asumido que una buena asistencia médica, odontológica y visual mejora la calidad de vida de las personas, pero se tiene poca evidencia ya que éstas son de tipo informal o anecdótico; con esta investigación se pretendió determinar si las percepciones de salud general, visual y oral influyen en la calidad de vida del adulto mayor para formular estrategias preventivas y programas que propicien los cambios y promuevan la cultura de la consulta oportuna, y garantizar que una población educada y atendida en promoción y prevención tendrá menos complicaciones de salud y por lo tanto menos costos de salud para la familia y el estado.

\section{MATERIALES Y MÉTODOS}

Se realizó un estudio descriptivo en el periodo de un año para analizar y explorar la relación de la percepción de salud con variables sociodemográficas de salud general, enfermedades crónicas así como factores orales y visuales, necesidad de tratamiento autopercibida y clínicamente percibida $\mathrm{y}$ visita profesional reciente o no.

Los datos se obtuvieron por encuestas aplicadas en la población adulta mayor que asiste a consulta en la Institución Prestadora de Servicios de Salud (IPS) de la Universidad Santo Tomás de Bucaramanga. La encuesta mencionada es una adaptación de la encuesta de salud de Cataluña de 1994 y el NANHES III y se validó por medio de una prueba piloto.

\section{Criterios de inclusión:}

- Ser mayor de 55 años de edad.

- Asistir a consulta externa en la IPS de la Universidad Santo Tomás, Bucaramanga.

- Querer participar en el estudio.

\section{Criterios de exclusión:}

- Estar incapacitado para responder la encuesta.

\section{Tamaño de la muestra:}

Se calculó según los siguientes datos:

En 1998, el total de pacientes adultos que asistieron a consulta de optometría fue de 628; según el estudio epidemiológico de la salud visual en la IPS, Clínica de Optometría en la Universidad. ${ }^{10} \mathrm{Al}$ tener en cuenta que se espera que el $50 \%$ de la población tenga algún tipo de percepción positiva o negativa, un margen de error del $5 \%$ y un nivel de confianza de $95 \%$ la muestra sería de $n$ $=165$ pacientes. Se recolectaron 136 encuestas de las que sólo fueron procesadas 106 por estar completas. Los participantes fueron elegidos de forma aleatoria según los criterios de inclusión; las encuestas fueron realizadas por los investigadores así mismo como la estandarización y la previa prueba piloto.

\section{Variables de estudio:}

A. Sociodemográficas: género, edad, municipio, barrio, ocupación, estrato social, hogar geriátrico, vive solo, seguridad social o SISBEN.

\section{B. Valoración médica}

1. Estado de salud general (expresa bienestar individual) si es positivo. Se valoró mediante la pregunta: ¿Usted diría que en general su salud es? Excelente, Muy buena, Buena, Regular, Mala. Las respuestas se agrupan para el análisis en: Buena salud o positivo (Excelente, muy buena y buena) y No Buena o negativo (Regular y mala). Además, se aplicó una escala análoga visual de nueve caras de McGrath, que muestran de sonriente a llorosa en escala de $1-10$ para determinar cómo su salud ha afectado su calidad de vida. ${ }^{11} \mathrm{Ne}$ cesidad de tratamiento o control auto percibida que se evaluó con la pregunta: ¿Cree usted que necesita asistir a consulta médica odontológica u optométrica para tratamiento o control? Visita profesional reciente esta variable se manejó de acuerdo a cada especialidad (medicina, odontología u optometría) y se clasificó como reciente si había acudido a ellas en el último año.

2. Discapacidades y enfermedades crónicas: incluye preguntas sobre limitación del habla, visión, escucha, escritura o lectura para salir, subir escaleras, actividades cotidianas, dependencia de equipos. Adicionalmente, pregun- 
tas sobre enfermedades crónicas sistémicas y toma de medicamentos, signos vitales y necesidad de tratamiento.

C. Valoración de salud visual. Se realizó un cuestionario para conocer las condiciones generales de salud visual y si había existido algún cambio de sus actividades debido a los problemas visuales. Además, se aplicó una escala análoga visual de nueve caras de McGrath, para determinar cómo su salud visual ha afectado su calidad de vida. ${ }^{11}$ Se indagó sobre rechazo a la realización de actividades de cerca o lejos, última visita al especialista en salud visual, actividades preventivas para el mejoramiento de su visión. Necesidad de tratamiento o control auto percibida se evaluó con la pregunta: ¿Cree usted que necesita asistir a consulta médica odontológica u optométrica para tratamiento o control? Visita profesional reciente esta variable se manejó de acuerdo a cada especialidad (medicina, odontología u optometría) y se clasificó como reciente si había acudido a ellas en el último año.

Examen clínico que estableció la necesidad de tratamiento clínico por el profesional de la salud por medio de un formato de tamizaje visual con agudeza visual, examen externo y valoración retinoscópica.

D. Valoración de salud oral. Incluyó cuestionamientos acerca de su condición general de salud oral. Además, se aplicó una escala análoga visual de nueve caras de McGrath, ${ }^{11}$ para determinar cómo su salud bucal ha afectado su calidad de vida: rechazo de alimentos por su condición oral, última visita al odontólogo o higienista, cuál fue la razón por la que lo hizo, asistió a chequeo o limpieza en los últimos tres años, y qué tan a menudo, necesita visitar algún odontólogo en especial, por cuánto tiempo ha estado en tratamiento regular, debe tomar líquidos para deglutir los alimentos, siente que su saliva es poca, tiene dificultad para deglutir algunos alimentos, siente sequedad cuando come un alimento o comida. Necesidad de tratamiento o control auto percibida que se evaluó con la pregunta: ¿Cree usted que necesita asistir a consulta médica odontológica u optométrica para tratamiento o control? Visita profesional reciente esta variable se manejó de acuerdo con cada especialidad (medicina, odontología u optometría) y se clasificó como reciente si había acudido a ellas en el último año.

Examen clínico que establece la necesidad de tratamiento clínicamente por el profesional de la sa- lud por medio un formato clínico así: Con un ICP, COP, CPTN y estado protésico, índice de higiene en prótesis y flujo salival estimulado considerado bajo 0-0,6; medio 0,6-1.0; alto 1.0-2.0. ${ }^{12-15}$

Consideraciones éticas: se tuvo en cuenta la resolución 008430 por la que se consideró un estudio sin riesgo, se elaboró el consentimiento informado y se aseguró la confidencialidad de los datos obtenidos. ${ }^{16}$

\section{Análisis de los datos:}

Inicialmente, se describieron las características sociodemográficas y las variables relacionadas con conocimientos y percepciones sobre la calidad de vida del adulto mayor. Posteriormente, según la naturaleza y distribución de las variables se aplicaron pruebas no paramétricas para comparar promedios para dos o más grupos, Kruskal Wallis para comparar las medianas de escala análoga visual, Las posibles asociaciones entre variables medidas en escala nominal y ordinal se evaluaron por medio de la prueba de $\mathrm{x}^{2}$ y test exacto de Fisher. Todo el análisis consideró un nivel de significancia $\alpha=0.05$, la base de datos se elaboró en Excel y el análisis definitivo en STATA 6.0.

\section{RESULTADOS}

De los 136 individuos participantes, sólo 106 encuestas pudieron ser procesadas e incluidas en el estudio por tener la información completa. La información se procesó con pruebas estadísticas no parametricas por la distribución de los datos.

La muestra estudiada estuvo compuesta por 46 individuos de género masculino y $60(56.6 \%)$ de género femenino, con edades que variaron entre 56 - 91 con una media de 68.6 años.

Cincuenta y una (48.1\%) personas procedían del área de Bucaramanga y 28 (26.4\%) de Floridablanca (Tabla 1). De los encuestados, tienen afiliación a seguridad social 53 (50\%) y SISBEN 42 (39.6\%). El estrato socioeconómico al que pertenecían se distribuyó principalmente en 1-2-3 con un 20\%, 25.4\% y $35 \%$, respectivamente. Veinte $(18.8 \%)$ de los participantes trabaja y los años de educación fueron en promedio 3.4 años. De la población estudiada, viven con su familia $83(78 \%)$ y $13(12.2 \%)$ viven en hogares geriátricos. 
Tabla 1. Características socio demográficas.

\begin{tabular}{lcc}
\hline \multicolumn{1}{c}{ Característica } & \multicolumn{2}{c}{ Frecuencia \% } \\
\hline \multirow{2}{*}{ Género } & Masculino & $46(43.4)$ \\
& Femenino & $60(56.6)$ \\
& Bucaramanga & $51(48.1)$ \\
& Floridablanca & $28(26.4)$ \\
Procedencia & Girón & $9(8.4)$ \\
& Piedecuesta & $6(5.6)$ \\
& Málaga & $1(0.9)$ \\
Seguridad social & Santa Bárbara & $4(3.7)$ \\
& Otros & $7(6.6)$ \\
& Seguridad social & $53(50.0)$ \\
& Sisben & $42(39.6)$ \\
Estrato socioeconómico & Ninguno & $6(5.6)$ \\
& Otras & $5(4.7)$ \\
& 1 & $21(19.8)$ \\
Vive con & 2 & $27(25.4)$ \\
& 3 & $37(34.9)$ \\
& 4 & $18(17.0)$ \\
Trabaja & 5 & $2(1.89)$ \\
& 6 & $1(0.94)$ \\
& Sí & $20(18.8)$ \\
& Nogar geriátrico & $86(81.8)$ \\
& & $83(78)$ \\
& & $10(9.4)$ \\
& Fomilia &
\end{tabular}

Población total: 106

Tabla 2. Frecuencia de enfermedades sistémicas.

\begin{tabular}{lc}
\hline \multicolumn{1}{c}{ Enfermedad Sistémica } & Frecuencia \% \\
\hline Diabetes & $10(9.43 \%)$ \\
Epilepsia & $1(0.94 \%)$ \\
Hipertensión & $44(41.5 \%)$ \\
Nervios & $15(14.1 \%)$ \\
Hemorragias & $4(3.77 \%)$ \\
Cardiovasculares & $12(11.3 \%)$ \\
Faringoamigdalitis & $10(9.43 \%)$ \\
\hline
\end{tabular}

Población total:106.

Tabla 3. Condición de salud general, oral y visual auto percibida y necesidades de tratamiento.

\begin{tabular}{|c|c|c|c|c|c|c|}
\hline \multirow{2}{*}{ Necesidad de Tratamiento } & \multicolumn{2}{|c|}{$\begin{array}{l}\text { Condición de salud general } \\
\qquad{ }^{*} \mathrm{P}=0.1\end{array}$} & \multicolumn{2}{|c|}{$\begin{array}{l}\text { Condición de salud bucal } \\
\qquad{ }^{*} \mathrm{P}=0.4\end{array}$} & \multicolumn{2}{|c|}{$\begin{array}{l}\text { Condición de salud visual } \\
\qquad{ }^{*} \mathrm{P}=0.6\end{array}$} \\
\hline & $\begin{array}{l}\text { Positiva } \\
\text { F } \%\end{array}$ & $\begin{array}{l}\text { Negativa } \\
\text { F \% }\end{array}$ & $\begin{array}{l}\text { Positiva } \\
\text { F\% }\end{array}$ & $\begin{array}{l}\text { Negativa } \\
\quad \text { F\% }\end{array}$ & $\begin{array}{l}\text { Positiva } \\
\text { F\% }\end{array}$ & $\begin{array}{l}\text { Negativa } \\
\quad \text { F\% }\end{array}$ \\
\hline $\begin{array}{l}\text { Necesidad de tratamiento } \\
\text { mediato }\end{array}$ & $55(66.2)$ & $28(33.7)$ & $39(46.9)$ & $44(53.0)$ & $21(46.6)$ & $24(53.3)$ \\
\hline inmediato & $1(100)$ & - & - & $1(100)$ & - & $1(100)$ \\
\hline Exámenes completos & $1(50)$ & $1(50)$ & - & $2(100)$ & - & - \\
\hline Remisión por hallazgo & - & $3(100)$ & $1(30)$ & $2(70)$ & - & - \\
\hline No necesita tratamiento & 9 (52.9) & $8(47.06)$ & $10(58.8)$ & $7(41.1)$ & $12(41.3)$ & $17(58.6)$ \\
\hline TOTAL & $66(62.2)$ & $40(37.7)$ & $50(47.1)$ & $56(52.8)$ & $33(44.0)$ & $42(56)$ \\
\hline
\end{tabular}

*Test exacto de Fisher
En relación con la condición de salud general, 33 (31.3\%) no reportaron enfermedad sistémica y 44 $(41.5 \%)$ registraron ser hipertensos. Se encontraron una o varias enfermedades por individuo con porcentajes reportados en la Tabla 2.

Sesenta y una (57.5\%) personas reportaron estar en tratamiento médico para su condición de salud y 59 (55.6\%) consumen medicamentos. Setenta y dos (67.9\%) reportaron no tener impedimento alguno y 18 (16.98) tienen impedimentos en la escucha. Se encontraron alteraciones en el pulso de $13(12.26)$ y presión en 42 (39.6\%); sin embargo, sólo 17 (16.3\%) creen necesitar tratamiento comparados con 57 (53.77\%) que tienen necesidades objetivas de tratamiento médico.

Sesenta y seis (62.26\%) de las personas descritas tenían una percepción positiva de su salud general y $40(37.7 \%)$ tienen una percepción negativa de su salud y han cambiado de actividades por culpa de ella 48 (46.09\%) (Tabla 3).

En la pregunta acerca de cómo su calidad de vida ha sido afectada por su salud general, en promedio fue de 3.07. Con respecto a la salud visual se encontró una percepción negativa de su salud visual en 56 (54.3) (Tabla 4). Cuarenta y cinco $(43.7 \%)$ individuos dejaron o cambiaron de actividades por su condición, 25 (24.2\%) nunca han visitado un optómetra y $37(35 \%)$ sólo lo visitan cuando es necesario; 62 (60.1\%) tienen o utilizan algún tipo de tratamiento visual.

La relación entre la escala análoga visual con su calidad de vida que se ha visto afectada por su salud visual, en promedio fue de 3.7.

De la misma forma se encontraron datos respecto a la salud bucal en la que la percepción de la salud fue positiva para $50(47.1 \%)$, y negativa para $56(52.8 \%)$ (Tabla 3). Cuarenta y tres $(40.2 \%)$ han dejado de consumir alimentos por su condición oral y 42 (39.6) 
tuvieron su última visita en los últimos seis meses, la razón principal en $58(54.7 \%)$ de ellos fue porque algo les molestaba, $74(69.8 \%)$ nunca se han realizado un tratamiento odontológico completo.

En la escala análoga visual para identificar qué tanto su calidad de vida ha sido afectada por su salud bucal, respondieron en promedio 3.6. De los participantes, sólo $39(36.8 \%)$ creen necesitar tratamiento y $89(83.96 \%)$ lo necesitan, realmente.

En el análisis bivariado se determinó cómo se ve afectada la calidad de vida de los adultos mayores (Tabla 4). Se evidenció que el factor que más la afecta es el dejar actividades tanto generales, dejar alimentos y visuales con $\mathrm{P}=0.0001$. Fue la percepción más negativa en cuanto tienen que dejar actividades con más frecuencia. Otros factores no mostraron diferencias estadísticamente significativas.

En la tabla 5 se muestran las percepciones de salud general, oral y visual, se observaron mayores percepciones negativas en salud visual y oral en las mujeres, en estrato 3 , aunque no se encontraron diferencias estadísticamente significativas para ninguna variable sociodemográfica.

El estrato socioeconómico no parece influir en gran manera las percepciones de la salud, sólo se observaron mayores percepciones negativas para estratos 1 y 2 en salud visual (Tabla 5).

La tabla 3 muestra cómo la percepción de la condición de salud tanto general, oral y visual sea positiva o negativa se relaciona con la necesidad objetiva de tratamiento. No se encontraron diferencias estadísticamente significativas pero se observaron percepciones positivas con necesidades de tratamiento en salud general y percepciones negativas en necesidades de tratamiento tanto odontológicas como optométricas y a pesar de no necesitar tratamiento se observan mayores cifras de percepción negativa para optometría.

Tabla 4. Factores que influencian la calidad de vida (medianas escala análoga visual).

\begin{tabular}{|c|c|c|c|c|c|c|}
\hline $\begin{array}{c}\text { FACTORES } \\
\text { Tiempo de consulta }\end{array}$ & $<6 \mathrm{~m}$ & $6-1$ año & 1 - 2 años & 2 - 3 años & Nunca & p \\
\hline General & 3.2 & 2.4 & 4.4 & 4 & 1 & ${ }^{*} 0.2$ \\
\hline Visual & 3.4 & 3.8 & 4 & 4 & 4.0 & ${ }^{*} 0.7$ \\
\hline Bucal & 3.6 & 3.4 & 4 & 3.3 & 3.7 & ${ }^{*} 0.7$ \\
\hline Razón visita & Chequeo & Algo molestaba & & & & \\
\hline General & 2.5 & 3.1 & & & & ${ }^{*} 0.2$ \\
\hline Visual & 3.0 & 4.2 & & & & ${ }^{*} 0.1$ \\
\hline Bucal & 3.7 & 3.3 & & & & ${ }^{*} 0.8$ \\
\hline Dejar actividades & Nunca & A veces & A menudo & Siempre & & \\
\hline General & 2.1 & 4 & 4.4 & 5 & & ${ }^{*} \mathrm{P}=0.0001$ \\
\hline Visual & 2.6 & 3.9 & 6.9 & 6.5 & & ${ }^{*} \mathrm{P}=0.0001$ \\
\hline Bucal & 2.4 & 5.3 & 5.4 & 4 & & ${ }^{*} \mathrm{P}=0.0001$ \\
\hline
\end{tabular}

*Test de Kuskal Wallis

Tabla 5. Percepción de salud y variables socio demográficas.

\begin{tabular}{|c|c|c|c|c|c|c|c|c|c|c|}
\hline \multirow{2}{*}{\multicolumn{2}{|c|}{ Percepción }} & \multicolumn{3}{|c|}{ General } & \multicolumn{3}{|c|}{ Visual } & \multicolumn{3}{|c|}{ Bucal } \\
\hline & & Positivo & negativo & $\mathbf{P}$ & positivo & negativo & $\mathbf{P}$ & positivo & negativo & $\mathbf{P}$ \\
\hline \multirow{2}{*}{ Género } & masculino & 32 (69.5) & $14(30.4)$ & & $24(52.1)$ & $22(47.8)$ & & $23(50)$ & $2350)$ & \\
\hline & femenino & 34 (56.6) & $26(43.3)$ & ${ }^{*} 0.1$ & $23(40.3)$ & 34 (59.6) & ${ }^{*} 0.2$ & $27(45)$ & $33(55)$ & ${ }^{*} 0.6$ \\
\hline \multirow{6}{*}{$\begin{array}{l}\text { Estrato } \\
\text { socioeconómico }\end{array}$} & 1 & 13 (61.9) & $8(38.1)$ & & $9(42.8)$ & $12(57.1)$ & & $12(57.1)$ & $9(42.8)$ & \\
\hline & 2 & 19 (70.3) & $8(29.6)$ & & $10(38.4)$ & $16(61.5)$ & & $13(48.1)$ & $14(51.8)$ & \\
\hline & 3 & $20(54)$ & 17 (45.9) & & $17(48.5)$ & $18(51.4)$ & & $16(43.2)$ & $21(56.7)$ & \\
\hline & 4 & $12(66.6)$ & $6(33.3)$ & & $9(50)$ & $9(50)$ & & $9(50)$ & $9(50)$ & \\
\hline & 5 & $1(50)$ & $1(50)$ & & $1(50)$ & $1(50)$ & & 0 & $2(100)$ & \\
\hline & 6 & $1(100)$ & 0 & ${ }^{*} 0.7$ & $1(100)$ & 0 & ${ }^{*} 0.8$ & 0 & $1(100)$ & ${ }^{*} 0.5$ \\
\hline TOTAL & & 66() & 40() & & 47 & 56 & & 50() & 56() & \\
\hline
\end{tabular}

*Test Exacto de Fisher 
Tabla 6. Relación de necesidades de tratamiento objetivas y subjetivas.

\begin{tabular}{|c|c|c|c|c|c|c|}
\hline \multirow{3}{*}{ Necesidad de tratamiento objetiva } & \multicolumn{6}{|c|}{ Necesidad de tratamiento subjetiva } \\
\hline & \multicolumn{2}{|c|}{$\begin{array}{l}\text { General } \\
{ }^{*} p=0.2\end{array}$} & \multicolumn{2}{|c|}{$\begin{array}{c}\text { Odontología } \\
* \mathrm{p}=0.02\end{array}$} & \multicolumn{2}{|c|}{$\begin{array}{l}\text { Optometría } \\
{ }^{*} \mathrm{p}=0.3\end{array}$} \\
\hline & no & si & no & si & no & si \\
\hline Necesidad de tratamiento mediato & $67(80.7)$ & $16(19.3)$ & $51(61.4)$ & $32(38.5)$ & $22(49)$ & $23(51)$ \\
\hline No necesita tratamiento & $17(100)$ & - & $15(88.2)$ & $2(11.7)$ & $18(62)$ & $11(38)$ \\
\hline
\end{tabular}

*Test exacto de Fisher

Tabla 7. Enfermedades sistémicas calidad de vida.

\begin{tabular}{lcccccc}
\hline \multicolumn{1}{c}{ Calidad de Vida } & \multicolumn{2}{c}{ General } & \multicolumn{2}{c}{ Visual } & \multicolumn{2}{c}{ Bucal } \\
& $\mathbf{~ m}$ & ${ }^{*} \mathbf{p}$ & $\mathbf{~ m}$ & ${ }^{*} \mathbf{P}$ & $\mathbf{m}$ & ${ }^{*} \mathbf{P}$ \\
\hline Diabetes mellitus & 2.8 & 0.9 & 4.8 & 0.4 & 4.6 & 0.7 \\
Epilepsia & 2 & 0.7 & 8 & 0.1 & 0 & 0.1 \\
Hipertensión & 3.3 & 0.3 & 4.4 & 0.05 & 3.8 & 0.4 \\
Nervios & 3.2 & 0.8 & 3.6 & 0.6 & 5 & 0.08 \\
Hemorragia & 5.7 & 0.05 & 6.6 & 0.006 & 7.5 & 0.01 \\
Cardiovascular & 5.1 & 0.006 & 5 & 0.20 & 4.3 & 0.3 \\
Faringoamigdalitis & 3.2 & 0.7 & 6.2 & 0.002 & 4.2 & 0.7 \\
\hline
\end{tabular}

*Test de Kruskal Wallis.

En la tabla 6 se compara la necesidad de tratamiento subjetiva o percibida por el paciente con las necesidades de tratamiento objetivas y se observó diferencias significativas para odontología, donde 51 (61.4\%) que necesitaban tratamiento consideraron no necesitarlo y $32(38.5 \%)$ con necesidad de tratamiento, sí consideraban necesitarlo con $\mathrm{P}=0.02$.

Dentro de las enfermedades sistémicas relacionadas con la calidad de vida autopercibida se observaron diferencias marginales en hemorragia y significativas en enfermedad cardiovascular y salud general con $\mathrm{p}=0.05$ y $\mathrm{P}=0.006$. La hipertensión se relacionó significativamente con la salud visual y calidad de vida con $\mathrm{p}=0.05$. Los nervios y hemorragias se relacionaron con la salud oral y calidad de vida (Tabla 7).

\section{DISCUSIÓN}

En este estudio se identificó la percepción que tienen los ancianos de la condición general, de su boca y de sus ojos y la influencia en su calidad de vida, con base en la conclusión de que la percepción general de salud es un predictor de la calidad de vida.

Este estudio reporta percepciones negativas de salud asociadas al género femenino para la salud vi- sual con 59.6\%, bucal con 55\% y general con un $43.3 \%$ así como lo destaca en su estudio Séculi y colaboradores en España donde se evaluaron 1459 varones y 1993 mujeres mayores de 60 años, el $57.3 \%$ de las mujeres declaró no tener una buena salud. ${ }^{6}$ Igualmente, Gallegos-Carrillo reportó percepciones negativas asociadas al género femenino con $66.7 \%$, estar incapacitado, enfermedades crónicas entre otras, en una población de 7322 adultos mayores de 60 años, en México. ${ }^{17}$

El mismo autor reporta que $14.72 \%$ de los individuos evaluados tenía limitación física y enfermedades crónicas como tensión arterial alta (33.6\%) y diabetes (17.1\%). Estos datos son similares a los encontrados en este estudio pues la tensión arterial alta ocupó un $41.5 \%$ de la población estudiada y la diabetes, un $9.4 \%$ asociados a percepciones negativas. La falta de ocupación reportada por Gallegos fue de $17.4 \%$ y para este estudio fue de $18.8 \%$.

La percepción positiva del estado de salud general para el presente estudio fue de $62.2 \%$ comparado con el estudio mexicano donde fue de $35.6 \%$; en un estudio realizado en Cataluña se observó un 45 a $55 \%$ entre diferentes grupos de edades. ${ }^{18}$ 
Vargas y colaboradores hacen algunas inferencias acerca de percepción negativa relacionada con nivel de riqueza en 1210 hogares, evaluaron un individuo por cada hogar; en este estudio no se encontraron asociaciones significativas para estrato socioeconómico. ${ }^{18}$

Respecto a la calidad de vida Aspiazu-Garrido y colaboradores encuestaron 911 individuos no institucionalizados y reportaron percepciones positivas de salud en $52 \%$ en adultos mayores sin diferencias al comparar con el género. ${ }^{19}$

Para calidad de vida, se encontraron algunas enfermedades sistémicas asociadas como hemorragias, enfermedades cardiovasculares y problemas visuales. Otros autores como Aspiazu-Garrido encontraron asociaciones con vivir solo; ${ }^{19}$ esta variable no fue significativa en este estudio.

En el análisis bivariado se encontró una asociación estadísticamente significativa para dejar actividades por causa de sus problemas de salud tanto general, visual y oral. Gallegos-Carrillo y colaboradores mencionan problemas visuales asociados a una mala percepción de salud, ${ }^{17}$ igualmente Stein y colaboradores encontraron individuos con peores percepciones de calidad de vida cuando sufren de degeneración macular asociada a la edad (ARMD) que cuando es leve la afección. ${ }^{20}$ Así mismo, este estudio encontró percepciones negativas en los aspectos visuales, principalmente.

Respecto a las percepciones de salud bucal, Jiménez y colaboradores encontraron percepciones más positivas en hombres y no encontraron asociaciones para el uso o no de prótesis total en su estudio sobre 80 ancianos. ${ }^{21}$ En este estudio se encontraron percepciones más negativas asociadas a necesidad de tratamiento odontológico pero sin asociaciones estadísticamente significativas.

Gift y colaboradores encontraron percepciones positivas de la condición de los dientes naturales asociada con necesidad de tratamiento subjetivo y otras variables de salud oral, sin embargo no encontró asociaciones con género y edad en un estudio realizado con 8377 personas de Estados Unidos. ${ }^{5}$

En este estudio, al relacionar la necesidad de tratamiento, la percepción de salud oral fue negativa en $53 \%$. En el estudio de McGrath y Bedi, el 61\% de los individuos consultó en el último año, 48\% consideró un efecto negativo de la salud oral en la calidad de vida. ${ }^{9}$ Los que consultaron en el último año creían que su salud oral mejoraba su calidad de vida (percepción positiva) con diferencias estadísticamente significativas; su estudio fue realizado en el Reino Unido con 1865 adultos, concluyeron que la atención regular mejora la calidad de vida.

En el presente estudio se encontraron percepciones negativas de calidad de vida asociadas a mayor tiempo de última visita odontológica, visual y general, sin diferencias significativas. Igualmente, sucedió para variables como motivo de consulta. McGrath y Bedi concluyeron en la importancia de la visita odontológica frecuente para mejorar la calidad de vida. ${ }^{7}$ Así mismo, Locker y colaboradores encontraron que la salud oral autopercibida como indicador de calidad de vida en diferentes modelos. ${ }^{22}$

Tovar, en el Valle (Colombia), encontró que el componente salud, muestra poca relación con la percepción; ${ }^{23}$ la salud individual, parece no influir de manera importante en la forma, en cómo se percibe; especialmente para salud general, probablemente porque el individuo se acostumbra y cree que estar enfermo es normal por ser viejo. Esto llama la atención, ya que aunque se trata de un auto-reporte, varios artículos reportan que el padecer enfermedades crónicas o una discapacidad fue el elemento que más influyó en el auto-reporte. Esta falta de correlación se debe probablemente a que la encuesta captura muy pocas condiciones de salud que si bien son importantes, no son suficientes para capturar el vínculo real que puede existir entre éstas y la percepción individual.

Para este estudio resultó que la condición que más afecta la calidad de vida es el tener que dejar actividades tanto por salud general, oral y visual. Las percepciones fueron más negativas en el género femenino.

\section{Conclusiones}

- Las percepciones negativas de calidad de vida fueron asociadas a mayor tiempo de última visita odontológica, visual y general sin diferencias significativas. Igualmente, sucedió para variables como motivo de consulta. 
- La condición que más afecta la calidad de vida es el tener que dejar actividades tanto por salud general, oral y visual.

- Las percepciones fueron más negativas en el género femenino.

- Para calidad de vida se encontraron algunas enfermedades sistémicas asociadas como hemorragias, enfermedades cardiovasculares y problemas visuales.

- Se encontraron percepciones más negativas asociadas a necesidad de tratamiento odontológico pero sin asociaciones estadísticamente significativas.

- Es necesario implementar actividades educativas que mejoren la asistencia periódica y frecuente a las consultas médicas, visual y odontológica como mecanismo de diagnóstico temprano y mayor asistencia a la consulta.

\section{Agradecimientos:}

A la Universidad Santo Tomás, seccional Bucaramanga por la financiación para la realización de este proyecto y su divulgación.

\section{BIBLIOGRAFÍA}

1. Cámara de comercio de Bogotá Colombia, siglo XXI, junio 1991.

2. Marín DJ. Es necesaria la odontogeriatria? Revista de la Federación Odontológica Colombiana 2000; 59.

3. República de Colombia. Departamento Administrativo Nacional de Estadística (DANE). Censo General, 2005 URL disponible en: www.dane.gov.co

4. Naciones Unidas. Comisión para el Desarrollo Social. Estrategia Internacional para la Acción, 2002 para el Envejecimiento. 29 de agosto de 2001 (adelanto de versión no publicada).

5. Gift HC, Atchison A, Drury TF. Perceptions of the natural dentition in the context of multiple variables. J Den Res 1998; 77: 1529 - 1538.

6. Sèculi E, Fuste.J, Brugulat P, Junca S, Guillen M. Percepción del estado de salud en varones y mujeres en las últimas etapas de la vida. Gaceta Sanitaria 2001; 15 : $217-223$.

7. Ettinger R. Oral disease and its effect on the quality of life. Gerodontics 1987: 3: 103 - 106.
8. Vargas CM, Kramarow EA, Yellowitz JA. The oral health of older Americans. Centers for disease Control and Prevention Aging Trends N3 March 2001.

9. McGrath C, Bedi R. Can dental attendance improve quality of life? Br Dent J 2001; 190: 262 - 265.

10. Rios H, Ayala M. Estudio epidemiológico de la salud visual en la IPS Clínica de Optometría USTA. 1998.

11. Quiles MJ, van-der Hofstadt CJ, Quiles Y. Instrumentos de evaluación de dolor en pacientes pediátricos: una revisión (2a parte) Rev Soc Esp Dolor 2004; 11: 360 - 369.

12. Camargo DM, Pieschacón MP. Acuerdo entre el índice de Green y Vermillon con el índice cuantificado de placa En: XI Encuentro de Investigación ACFO, Memorias. División de Investigación ACFO. Facultad de Odontología, CES Medellín 2000 p. 57.

13. República de Colombia. Ministerio de Salud. Discusión general de promoción y prevención. III Estudio Nacional de Salud Bucal y II Estudio de Factores de Riesgo de las Enfermedades Crónicas. Bogotá: Ministerio de Salud; 1999. p. $69-153$.

14. Concha SC. Análisis de la asociación entre la calidad vida y la condición oral de las personas mayores vinculadas a Instituciones geriátricas de Bucaramanga: II fase. XVIII Encuentro Nacional de Investigación Odontológica ACFO. II Reunión de la International Association of Dental Research Manizales UAM- ACFO 2007.

15. Marques Soares MS. Estudio clínico de pacientes con Síndrome de Boca Ardiente: xerostomía, flujo salival, medicamentos, ansiedad y depresión. Tesis Doctoral, Universidad de Barcelona; 2006.

16. República de Colombia. Ministerio de Salud. Resolución 008430 de 1993. URL disponible en: www.ins.gov.co/pdf admon/juridica/ei/ei 1993 res ms 8430.pdf

17. Gallegos-Carrillo K, Garcia-Peña C, Duran-Muñoz C, Reyes H, Duran-Arenas L. Autopercepción del estado de salud: una aproximación a los ancianos en México. Rev Saúde Pública 2006; 40: 792 - 801.

18. Vargas A, Gutiérrez JP. Percepción de los estados de salud en la República Mexicana URL disponible en: www.abres.cict.fiocruz.br/trabalhos/mesa11/4.pdf

19. Azpiazu Garrido M, Cruz Jentoft A, Villagrasa Ferrer J R, Abanades Herranz C, García Marín N, Valero FA. Factores asociados a mal estado de salud percibido o a mala calidad de vida en personas mayores de 65 años. Rev Esp Salud Pública 2002; 76: 683 - 699.

20. Stein JD, Brown MM, Hollands H, Sharma S. Quality of life with macular degeneration: perceptions of patients, clinicians, and community members. Br Ophthalmol 2003; 87: $8-12$.

21. Jiménez J, Esquivel RI, González-Celis RAL. La percepción de la salud bucal como medida de calidad de vida en ancianos. ADM 2003; 60: $19-24$.

22. Locker D, Clarke M, Payne B. Self-perceived oral health status, psychological well being, and life satisfaction in an older adult population. J Dent Res 2000; 79: 970 - 975.

23. Tovar LM. Determinantes del estado de salud de la población colombiana. Econ Gest Desarro 2005; 3: 125 151. URL disponible en: http://revistaeconomia.puj.edu. co/revista_3/6.pdf 